\title{
Le Prix du Livre Européen: promoteur et relayeur des liminalités littéraires ${ }^{1}$
}

José Domingues de Almeida

Université de Porto - ILC

Résumé: Cet article se penche sur la pertinence de l'attribution d'un prix littéraire tel que Prix de Livre Européen pour la promotion d'une instance de légitimité littéraire européenne, pour l'affirmation de l'adhésion à l'esprit de l'UE, ainsi qu'au dégagement de certaines constantes thématiques : liminalités, postmémoire, interculturalité, etc.

Mots-clés: prix littéraire, liminalités, Europe

Abstract: This paper examines the relevance of awarding a literary prize such as the European Book Prize for the promotion of a body of European literary legitimacy, for the adherence to the spirit of EU membership, as well as the identification of certain thematic constants: liminalities, postmemory, interculturality, etc.

Keywords: Literary prize, liminalities, Europe

Le Prix de Livre Européen a été créé en 2007 par l'association “Esprit d’Europe”, avec le soutien de Jacques Delors, premier président du comité de parrainage jusqu'en 20II, à qui a succédé Pascal Lamy. Cette association, présidée par France Roque, organise depuis lors chaque année la remise de cette récompense littéraire ( 10.000 euros) en début décembre, au Parlement européen à Bruxelles. Le prix récompense un roman et un essai exprimant et promouvant une vision positive de l'Europe. 
C'est dire combien notre continent aurait terriblement besoin de motivation et de mobilisation, et combien le "projet européen" demeure vague et dans une certaine mesure, artificiel et éloigné des tout premiers soucis identitaires des peuples qui constituent l'Union européenne, comme le prouve à l'envi le taux élevé d'abstention aux élections européennes. Ceci révèle un insurmontable décalage entre l'approche du Vieux Continent en tant que matrice civilisationnelle et horizon géosymbolique, d'une part, et l'Union européenne en tant que construction politique et institutionnelle, en tant que constructus, qui mène au besoin de créer de toutes pièces des attaches à l'“esprit” européen.

C'est justement le cas de la création de ce prix littéraire, mais c'est tout aussi bien la même logique qui préside aux outils promotionnels d'un sentiment d'Europe ("européanité") par l'investissement institutionnel dans des programmes que d'aucuns qualifient d'“idéologiques" (Maurer 20II) tels le Cadre Européen Commun de Référence pour les Langues, le programme de mobilité étudiante et enseignante Erasmus, voire la création d'une monnaie unique et la tentation d'un fédéralisme institutionnel, le tout pour contrecarrer un europessimisme endogène dont le brexit est le dernier avatar.

Autrement dit, les Européens impliqués institutionnellement dans l'Union européenne rechignent encore à sacrifier une part de leur souveraineté nationale, ou à se sacrifier tout court en faveur de l'intégration dans un empire consenti. En effet, la création du Prix du Livre Européen coïncide avec des phénomènes inquiétants et facilement subversifs des idéaux européens: la crise (im)migratoire et les échecs de l'intégration/ assimilation et de la coexistence communautaires dans certaines métropoles européennes, la montée des extrémismes, des radicalismes et des intégrismes, l'ankylose de l'Europe en tant que “projet”, laquelle va de pair avec les interrogations autour de la notion d'identité nationale et, de surcroît, européenne.

C'est dire combien la création d'une instance de légitimité littéraire au niveau européen est idéologiquement motivée, régie par des critères de promotion de sentiments forcément "positifs" de l'Europe, souvent dans la doxa eurocratique qui brasse les concepts parfois euphémistiques d'inclusion ( $v s$ assimilation), migration ( $v s$ immigration clandestine), résilience ( $v s$ résistance) ou durabilité ( $v s$ politique). Disons qu'elle est en phase avec la logique du serpent qui se mord la queue du financement des projets scientifiques européens, relayé par les États-membres, puisque des fonds européens financent des thématiques privilégiant la doxa et les soucis européens. Citons-en deux où nous sommes impliqués: le partenariat stratégique de plusieurs universités au sein du projet piloté par Budapest (K4203) en articulation avec LÉA (Lire en Europe aujourd'hui) et le Projet stratégique ILCML: “Littérature et Frontières de la Connaissance: politiques d'inclusion”, dont une des lignes subsidiaires concerne les “nouvelles figurations du discours sur l'Europe”. Rappelons aussi que l'ILC (Instituto de Literatura Comparada) a explicitement créé une base de données, “L’Europe 
face à l'Europe" à deux catégories (poètes et prosateurs) dont la construction est en cours, et dont les entrées pointent des représentations du Vieux Continent dans, et par le fait littéraire. ${ }^{2}$

Dans le même sens, on peut considérer l'initiative du "Hay Festival", un festival littéraire annuel qui se produit au Pays de Galles et qui en 2019 s'ouvre à une relecture de l'avenir de l'Europe repensé par vingt-huit auteures, représentantes de chacun des États-membres par le biais de la fiction, du conte et de l'essai, laquelle donnera lieu à une anthologie intitulée Europe28: Visions pour l'Avenir qui sera lancée à Rijeka (Croatie), capitale européenne de la culture 2020. L’auteure portugaise de contes Ana Pessoa y représente le Portugal, alors que Leïla Slimani est l'écrivaine française retenue. Les vingt-huit contributrices sont censées exprimer ou évoquer ce que l'Europe signifie pour chacune d'elles par le conte ou l'essai. ${ }^{3}$

Mais revenons sur le Prix du Livre Européen. Pour ce qui est de la méthodologie d'attribution, notons que les auteurs des deux ouvrages (roman et essai) sont des écrivains contemporains originaires de pays membres de l'Union européenne. Parmi les livres correspondant à ces critères et publiés dans l'année par les maisons d'édition de chacun des vingt-huit pays membres de l'Union européenne, une présélection est soumise au Comité de parrainage. Celui-ci détermine ensuite une sélection définitive, composée d'une dizaine d'ouvrages (cinq essais et cinq romans) qui sont ensuite présentés au jury, présidé en 2018 par le metteur en scène polonais Krzysztof Warlikowski. La cérémonie de remise du prix se tient au Parlement européen, à Bruxelles.

Or l'attribution et la divulgation médiatique d'un tel prix entraînent des conséquences commerciales et éditoriales: renforcement des ventes, relai de la critique, présence médiatique, circulation des œuvres sur le territoire européen, notamment par le biais de la traduction, et souvent à partir des langues minoritaires vers les langues européennes de plus large diffusion et au marché éditorial plus important, comme l'anglais ou le français. C'est d'ailleurs à partir de la traduction française de ces textes que nous nous pencherons sur les thématiques liminales saluées et retenues dans la présélection et chez les lauréats du Prix du Livre européen dans la catégorie "roman".

En effet, une lecture attentive des titres nommés ou vainqueurs, et ce à partir d'un échantillon, ${ }^{4}$ fait apparaître la récurrence de constantes thématiques plus ou moins explicites, mais qui se regroupent toujours autour de l'axe européen qui a motivé leur sélection et dont nous retiendrons, sans les systématiser: les post-mémoires nationales dans le concert européen, les liminalités et la création de nouveaux imaginaires frontaliers, l'entre-deux / la mitoyenneté géo-symbolique et la cohabitation ou confrontation culturelle.

Notre approche de l'Europe littéraire s'inscrit, par ailleurs, dans un contexte où la recherche en littérature a pris acte de la crise de la théorie littéraire après les an- 
nées 1970 dans ses différentes mouvances: sémiotique, poststructuraliste, marxiste et psychanalyste, et privilégie de nouvelles approches: études postcoloniales, ethnicité, sexualité / genre et études culturelles, ce qui dessine de facto un tournant du discours (idée) vers la culture (réel) (Moura 2018: 104), lesquelles coïncident dernièrement avec une "pensée géographique de la littérature” (idem: 107) où prennent part justement les “études régionales” (area studies).

Cette réorientation des outils théoriques de la recherche en littérature n'est pas sans rapport avec l'infléchissement causé par la perspective du “mondial” ou du "global” sur la littérature comparée (Spivak 2003), à laquelle d’aucuns reprochent, outre le déséquilibre entre théorie et application, l'indéfinition méthodologique et son occidentalo-centrisme, la quasi exclusive référence au national (Moura 2015). Or la promotion critique d'une perspective régionale et spatiale du fait littéraire (l'Atlantique, la Méditerranée, l’Europe, etc.) coïncide avec le déclin des compétences linguistiques pour lire l'original et, partant, avec un regain d'importance de la littérature traduite.

De ce fait, l'Europe littéraire en perspective - qui plus est relayée par une instance de légitimation et d'institutionnalisation (Dubois [1978] 2005), ledit Prix du Livre européen - suscite de nouveaux sujets et topiques (postcoloniaux) dans l'imaginaire européen, rend l'Europe sous un jour postcolonial (Schulze-Engler 2013), réécrit la ville / métropole européenne, la “ville-monde" (Zemmour 2014: 446-470), la cité globale (Sassen 200I) à partir d'une perspective migrante, diasporique ou mobile; investit des lieux non-métropolitains, sans implication directe dans le colonialisme, comme les espaces ruraux, le terroir, les îles, etc. lesquels accèdent à un imaginaire littéraire européen.

Aussi, s'il est possible de lire dans certains textes fictionnels un phénomène de “provincialisation” de l'Europe à partir du dehors, d'un regard exotopique postcolonial sur le Vieux Continent (Chakrabarty 2000), comme dans Alentejo blue (Café Paraíso pour la traduction française d'Isabelle Maillet) de Monica Ali (2007), Anglaise originaire du Bengladesh, dont le récit choral retrace plusieurs strates de l'existence dans un village imaginaire de l'Alentejo (Portugal) baptisé Mamarrosa; ou encore le roman Un rêve utile de Tierno Monénembo (2007), lequel transpose carrément une société africaine dans une ville française imaginaire nommée Loug, où l'on retrouve subtilement l'étymon Lugdunum (Lyon), c'est en raison de la réévaluation des espaces périphériques du continent européen où s'opère un réaménagement des rapports entre marges et centre, (Peeren, Stuit et Van Weyenberg 2016; Ameel, Finch et Salmela 2015), voire une redécouverte de la province européenne, ou un regard nouveau sur les centres métropolitains perçus comme lieux de transculturation et de cosmopolitisme.

Le Prix du Livre européen prime, lui aussi, de telles perspectives en signalant à partir d'une démarche de légitimation institutionnelle de l'Union européenne des aspects géo-historico-culturels d'un continent bien plus vaste que cet ensemble 
politique. C'est le cas, par exemple, du récit primé en 2017, Zinc de l'écrivain et activiste belge David Van Reybrouck (2017). Il s'agit là d'un documentaire fictionnalisé, mais aussi utopique, qui a pour sujet l'originale autogestion dans un espace et un temps bien déterminés (les deux Grandes guerres) à Moresnet-Neutre, petite commune germanophone annexée par la Belgique au Traité de Versailles, mais dont le statut autonome fut respecté jusque-là par les trois États limitrophes. Bien évidemment, ce village métaphorise une certaine idée de l'Europe frontalière, de région liminale et des confins entre les domaines germanique et latin de l'Europe.

La neutralité de Moresnet-Neutre, maintenue entre 1816 et 1919, grâce à la présence d'une ancienne mine de zinc dans le village (La Calamine), enviée par les États limitrophes, permet ainsi d'illustrer des théories politiques de démocratie directe ou de gouvernance locale improvisée prônée par Van Reybrouck: "Le compromis consistait en une absence de compromis (...). Cette zone devenait territoire neutre” (2017:18).

Le récit retrace le parcours historique d'Emil, enfant adultérin d'une domestique allemande chassée de chez ses employeurs au début du XXe siècle, qui se réfugie dans le sanctuaire neutre de Moresnet-Neutre, ce qui en fait un emblème d'un pan de l'histoire de notre continent:

Sans avoir déménagé une seule fois de sa vie, il a été successivement citoyen d'un État neutre, sujet de l'Empire allemand, habitant du royaume de Belgique et citoyen du Troisième Reich. Avant de redevenir belge, ce qui sera son cinquième changement de nationalité, il [Emil] est emmené comme prisonnier de guerre allemand. Il n’a pas traversé de frontières, ce sont les frontières qui l’ont traversé. (idem: 63)

Dans le même sens, on peut lire le roman de la lauréate finlandaise du prix en 2010, Sofi Oksanen, Purge. Dans cette fiction, la romancière renoue avec son activisme contre toutes formes de totalitarisme, ressasse l'identité des pays baltes (Estonie-Finlande), s'attaque au mythe de la "finlandisation" en interrogeant les clichés de la culture et de l'identité finlandaises - cette idée construite et entretenue d'une société pacifique et exceptionnellement heureuse -, et exhume les crimes idéologiques commis sur ces territoires à la faveur des changements successifs de régime: nationalisme balte, national-socialisme et communisme, pour en arriver à l'intégration européenne.

C’est d'ailleurs ce que représente et synthétise le parcours historique personnel du personnage de la vieille Aliide, à savoir un regard sur la mémoire de cette liminalité géographique du Vieux Continent (grand nord, baltique) qui apparaît, certes, comme périphérique, mais fortement connectée aux déboires causés par les événements qui ont marqué l'Europe au XXe siècle. Dans Purge, nous suivons le parcours tragique d'une famille sur trois générations de femmes imbriquées et impliquées sous plusieurs régimes politiques successifs, avec leur lot d'espoirs, d'arrangements, 
de compromissions, mais surtout de trahisons et de délations, le tout dans un incessant va-et-vient historique fait d'analepses et de prolepses qui expose différentes tranches historiques:

Les yeux de sa grand-mère ont brillé lorsque Zara lui a annoncé son intention d'aller travailler en Allemagne. Sa mère n'en avait pas été enthousiasmée, rien ne l'enthousiasmait d'ailleurs, mais surtout, elle n'aimait pas ces projets, car elle pensait que l'Ouest était un endroit dangereux. Un salaire élevé n'avait pas changé l'opinion de sa mère. Sa grandmère elle-même n'avait pas fait attention aux conversations de Zara sur l'argent, mais elle avait exigé que, avec cet argent, Zara aille visiter l'Estonie. (2011: 03)

Mais le Prix du Livre Européen a surtout récompensé des retours postmémoriels sur l'histoire européenne, aux conséquences somme toute assez récentes. Rappelons que le concept de "postmémoire" définit, selon Marianne Hirsch:

(...) la relation que la "génération d’après" entretient avec le traumatisme personnel, collectif et culturel subi par ceux qui l'ont précédée, avec des expériences dont elle ne "se souvient" que par le biais d'histoires, d'images et de comportements au milieu desquels elle a grandi. Mais ces expériences lui ont été transmises si profondément et avec tant d'émotion qu'elles semblent constituer une mémoire en tant que telle. (2008: 114)

Si, bien souvent, ce rapport complexe au passé s'inscrit du côté des descendants des victimes, c'est-à-dire la troisième génération de la famille des déportés (Rubinstein 2002; Skowronek 2015), une fois la Shoah entrée dans le domaine public, la récompense en question a salué le même vécu du côté des descendants des bourreaux, des collaborateurs, ou tout simplement des "suiveurs" sympathisants. C'est le cas de deux récits plus ou moins fictionnels, écrits par deux auteurs, symptomatiquement produits sur l'axe franco-allemand, primés par le Prix du Livre Européen, respectivement Histoire d'un Allemand de l'Est de l'écrivain et journaliste Maxim Leo (2010), lequel fait contraster le sort de ses deux grands-pères, l'un communiste persécuté sous le national-socialisme et l'autre, admirateur du Ille Reich, et la lauréate du Prix 2018, Géraldine Schwarz, pour Les Amnésiques, où sont implacablement abordés, à partir de l'histoire familiale de l'auteure, les thèmes de la confiscation des biens de propriétaires juifs, notamment les entreprises, au profit des Allemands (et notamment le grand-père de l'écrivaine), celui encore tabou de la collaboration passive (mitläufer), et partant, de la mauvaise conscience de bien des peuples européens sous le joug allemand:

Il est impossible que mes grands-parents aient ignoré la propagande antisémite qui inondait les ondes radiophoniques, les journaux et les pancartes dans les espaces pu- 
blics. Eux qui étaient si entourés, n’avaient-ils jamais entendu raconter que tel médecin, avocat, fonctionnaire s'était retrouvé à la rue après des années de loyaux services? Ou comment une mère de famille avait vu soudain l'école de ses enfants chasser une partie de ses élèves, exclus parce que juifs? (Schwarz 2015: 195)

Par ailleurs, le Prix du Livre Européen a parfois mis en avant les aspects géographiquement liminaux de l'Europe en les accouplant d'une réflexion sur la mobilité intra-européenne, ainsi que sur les heurts et incompréhensions interculturels sentis par les Européens. C'est le cas de Les Complémentaires de l'écrivain danois Jens Christian Grøndahl ([2013], 2015], présélectionné en 2015, et qui renvoie à une certaine image du continent européen dans son rapport centre-liminalité (nordique) et à sa composition multiculturelle, parfois problématique, surtout dans les grandes métropoles, la "ville-monde" de Londres. En tous cas, la fiction grondahlienne circule en Europe et thématise subtilement ce contient. Tout comme pour Oksanen, il n'est pas sans intérêt de relever que l'assignation liminale imaginaire nordique, qui impliquait souvent l'assimilation au cliché générique du polar scandinave, oblige désormais à des nuances, pour dégager un roman nordique non policier. ${ }^{5}$ Dans ce roman-ci par exemple, la mobilité Londres-Copenhague est productrice de confrontations et de difficile coexistence ou intégration dans un continent où le communautarisme commence à faire rage:

Il fut surpris de l'[sa belle-mère] entendre radoter ainsi sur les musulmans. Il était étonné car, durant toutes ces années, il l'avait soupçonnée de nourrir un petit peu d’antisémitisme, comme un animal de compagnie, tout au fond du sous-sol muet de sa personnalité. Et pourquoi sa phobie de l'Islam n'y aurait-elle pas fleuri elle aussi? Était-ce sa paranoïa latente à lui qui le faisait s'étonner du fait que, avec son antisémitisme caché, elle n'éprouvait pas le besoin de fêter les Arabes qui, eux, haïssent les Juifs de manière machinale? (Grøndahl 2015: 30)

Mais derrière le rituel de l'attribution d'un prix comme celui qui nous occupe ici, et dont nous ne nous penchons que sur certains aspects et par un échantillon assez limité, est engagé tout un circuit commercial, même si la récompense n’a certes pas l'impact d'autres trophées littéraires à l'étranger par le biais de la traduction, même décernés en France, tels les prix Goncourt ou Femina. C'est dire l'importance dont se revêt la traduction et le rôle incontournable du traducteur littéraire dans la médiation des ouvrages. D’autant plus que bien des romans et récits primés nous arrivent en français par la traduction directe, rarement indirecte, dont il nous est force de relever souvent la qualité, mais aussi moyennant la traduction indirecte, notamment de langue européenne minoritaire vers une autre langue de moindre circulation. C'està-dire que les textes connaissent parfois un circuit périphérie-centre-périphérie; une 
pratique courante de circulation des textes fictionnels en Europe, mais qui met à nu le peu de relief donné au traducteur dans cette même circulation, lequel peut apparaître comme le grand oublié.

Ceci dit, et pour conclure, l'institution d'un prix littéraire tel que le Prix du Livre Européen, même si elle n'est pas en soi directement créatrice de liminalités linguistiques, géographiques, imaginaires ou fictionnels, n'en est pas moins promotrice et relayeuse de ces réalités européennes dans une logique qui produit la circulation des ouvrages, et ce faisant, des fictions. À cet égard, elle s'avère opportune. Les questions soulevées par la création littéraire rendent souvent l'Europe dans ses complexités, bien au-delà d'une vision purement "positive" de notre continent, si l'on confère à cette épithète le sens de "favorable" ou "promotionnel”. En fait, les ouvrages primés ou sélectionnés véhiculent des images nuancées, et souvent complaisantes, de nos sociétés et de leurs histoires et conflits plus ou moins récents. En ce sens, cette récompense s'avère "positive" et peut sans doute contribuer à une relecture de nos identités composites au sein du projet européen en cours.

\section{Notes}

* José Domingues de Almeida est Maître de Conférences à la Faculté des Lettres de l'Université de Porto. Il est docteur en littérature francophone contemporaine. Ses domaines de recherche sont la littérature française contemporaine, les études francophones et la culture et pensée françaises contemporaines. Il se penche récemment sur les questions théoriques et critiques soulevées par les littératures post-migratoires, les récits post-mémoriels et les représentations de l'Europe. Il est chercheur à l'Instituto de Literatura Comparada Margarida Losa et directeur de la revue électronique Intercâmbio. Il est, par ailleurs, vice-président de l’Association Portugaise d’Études Françaises.

' Le présent article s'insère dans la recherche menée dans le cadre du Projet de l'Institut de Littérature Comparée, financé par la Fondation pour la Science et la Technologie (UIDB/00500/2020).

${ }^{2}$ Son projet se décline comme suit sur le site de la base: "Quels dialogues la littérature, en elle-même et en rapport intertextuel avec d'autres arts, établit-elle avec l'Europe post-1945? De quelle façon l'écriture interroge-t-elle l'identité et les frontières d'un continent pluriel, simultanément très ancien et en constante réinvention, notamment ces 70 dernières années? Quelles aspirations, interrogations, protestations continuent de décrire et forcent à re-décrire, ouvrage à ouvrage, l'histoire et la confluence de civilisations, l'inclusion et l'exclusion de peuples? De quelle façon, enfin, la création artistique 
réinvente-t-elle l'Europe même en proposant de nouveaux modèles d'échange d'une expérience?". http://aeuropafaceaeuropa.ilcml.com/fr/

${ }^{3}$ cf. https://expresso.pt/cultura/2019-06-12-Autora-portuguesa-Ana-Pessoa-e-uma-das-28-artistas-escoIhidas-para-repensar-o-futuro-da-Europa

${ }^{4}$ Notre échantillon prend surtout pour point de départ notre propre contribution (entrées) à la construction de la base de données L'Europe face à l'Europe. http://aeuropafaceaeuropa.ilcml.com/fr/les-prosateurs-ecrivent-leurope/

${ }^{5}$ https://observador.pt/especiais/sofi-oksanen-nao-existe-lugar-para-os-livros-nordicos-que-nao-sao-policiais/

\section{Références bibliographiques}

Ali, Monica (2007), Alentejo Blue, trad. de Manuel Valle Cintra. Lisboa, Dom Quixote. Ameel, Lieven, Finch, Jason et Salmela, Markku (Eds.) (2015), Literature and the Peripheral City. NY, Springer.

Chakrabarty, Dipesh (2000), Provincializing Europe. Princeton, Princeton University Press.

Dubois Jacques (2005), L'Institution de la littérature. Bruxelles, Labor.

Grøndahl, Jens Christian (2013 [2015]), Les Complémentaires. Paris, Gallimard, coll. «Folio».

Hirsch, Marianne (2008), “The generation of postmemory”, Poetics Today, vol. 29, no I, pp. I03-I28.

Leo, Maxim (2010), Histoire d'un Allemand de l'Est, Paris, Arles, Actes Sud, coll «Babel».

Maurer, Bruno (2011), Enseignement des langues et construction européenne. Le plurilinguisme, nouvelle idéologie dominante. Paris, France, Éditions des Archives Contemporaines.

Monénembo, Tierno (2007), Un rêve utile. Paris, Seuil.

Moura, Jean-Marc (2015), L’Atlantique littéraire. Perspectives théoriques sur la construction d'un espace translinguistique. avec Véronique Porra, Hildesheim, Olms Verlag.

Moura, Jean-Marc (2018), “Un nouvel espace pour l'histoire littéraire comparatiste: l'Atlantique”, Nouveaux mondes, nouveaux romans, Société Française de Littérature Comparée,pp.ı4-I09, accessible sur http://sflgc.org/acte/jean-marcmoura-un-nouvel-espace-pour-lhistoire-litteraire-comparatiste-latlantique/

Oksanen, Sofi (2008 [20II]), A Purga. Carnaxide, Editora Objetiva. 
Peeren, E., Stuit, H. et Van Weyenberg, A. (Eds.) (2016), Peripheral Visions in The Globalizing Present, Space, Mobility, Aesthetics. Leide, Boston, Brill.

Rubinstein, Marianne (2002), Tout le monde n'a pas la chance d'être orphelin. Paris, Verticales.

Sassen, Saskia (200I), The Global City. Princeton, Princeton University Press.

Schwarz, Géraldine (2017), Les Amnésiques. Paris, Flammarion.

Schulze-Engler, Frank (2013), “Irritating Europe”, in Graham Huggan (Ed.), The Oxford Handbook of Postcolonial Studies. Oxford, Oxford UP, pp. 669-69I.

Skowronek, Nathalie (2015), La Shoah de Monsieur Durand. Paris, Gallimard.

Spivak, Gayatri Chakravorty (2003), Death of a Discipline. NY, Columbia University Press.

Van Reybrouck, David (2016), Zinc. Arles, Actes Sud.

Zemmour, Éric (20।4), Le Suicide français. Paris, Albin Michel.

\section{Sitographie:}

https://observador.pt/especiais/sofi-oksanen-nao-existe-lugar-para-os-livrosnordicos-que-nao-sao-policiais/ [disponible le 19/12/20]

http://aeuropafaceaeuropa.ilcml.com/fr/les-prosateurs-ecrivent-leurope/ [disponible le 19/12/20]

https://expresso.pt/cultura/2019-06-12-Autora-portuguesa-Ana-Pessoa-e-uma-das28-artistas-escolhidas-para-repensar-o-futuro-da-Europa [disponible le 19/12/20] 